\title{
New preformed catheter for entry into pulmonary artery in complete transposition of great arteries
}

\author{
Fergus J. Macartney, Olive Scott, Philip B. Deverall, and Fred Hepburn \\ From the Department of Paediatric Cardiology, Killingbeck Hospital, Leeds
}

A new preformed, semi-rigid, polyethylene catheter, with built-in torque control, has been devised for entry to the pulmonary artery in complete transposition of the great arteries. It has been used I9 times in I7 patients: 18 times the pulmonary artery was entered from the right atrium (via the left atrium and ventricle) in a time between $40 \mathrm{~s}$ and $15 \mathrm{~min}$ (median $5 \mathrm{~min}$ ); the patients' ages were 2 days to 6 years (median 8 months) and their weights were 3.1 to $13.3 \mathrm{~kg}$ (median $6.9 \mathrm{~kg}$ ); in the remaining I day-old patient, the procedure was terminated because of atrial flutter.

The catheter was introduced into the axillary vein in 3 patients and the saphenous or femoral vein in the remainder. It is suitable for angiocardiography, and the other heart chambers and vessels were easily entered.

Thus the catheter has certain advantages over previously described methods for entry to the pulmonary artery, particularly when there is inferior vena caval thrombosis, or when angiocardiography is necessary. Its use does depend on the presence of an interatrial communication, so a method for entry to the pulmonary artery by retrograde catheterization from the axillary artery using a different catheter is also presented; this was successful in 2 patients with ventricular septal defect.

It is now eight years since the first papers on entry into the pulmonary artery in transposition of the great arteries were published (Carr and Wells, 1966; Rahimtoola, Ongley, and Swan, 1966). Since then at least five other methods for entering the pulmonary artery have been described (Celermajer, Venables, and Bowdler, 1970; Kelly, Krovetz, and Rowe, 1971 ; Pickering, McDonald, and Kidd, I97I ; Tynan, 1972; Mullins et al., 1972; Stanger et al., 1972; Black, 1972; Jones and Miller, 1973). The major disadvantage of most of these is that the catheter used is not suitable for angiocardiography. Since speed in cardiac catheterization of sick infants undoubtedly reduces the mortality of the procedure, an ideal catheter would be one that would invariably reach the pulmonary artery, but could also be used for entry into other chambers and vessels, for sampling, and for angiocardiography.

The new catheter to be described goes some way towards achieving this ideal, but does depend on the presence of an interatrial communication. For this reason a variant of the retrograde arterial method (Mullins et al., 1972), using a different catheter, useful for patients with ventricular septal defect but intact atrial septum, is also described.

Received 28 October 1974 .

\section{Subjects and methods}

Before July 1972 a variety of different methods had been used at this institution to enter the pulmonary artery in complete transposition with an overall 16 per cent failure rate. At this point the Cobra catheter, ${ }^{1}$ originally designed for selective abdominal arteriography, was first used for this purpose and found to be surprisingly successful. A prospective study of its use was then set up, simply by measuring the total time taken for the catheter to enter the pulmonary artery from the right atrium with a stop-watch. In patients with ventricular septal defect an attempt was first made to enter the pulmonary artery anterograde through the ventricular septal defect with a conventional catheter, since this is successful in our hands in 64 per cent of patients. In all other patients, the new catheter was used as the method of first choice in all patients with complete transposition of the great arteries except for two (v.i.) in whom there was strong evidence from previous catheterization that the interatrial septum was intact (this was subsequently confirmed at operation).

Our experience before the introduction of this catheter was that the interests of the sick, severely cyanosed infant with transposition were best served by balloon atrial septostomy immediately the diagnosis had been established and return to the intensive care unit as soon as possible after adequate septostomy had been achieved.

\footnotetext{
${ }^{1}$ Cordis Corp., Miami, Fla., U.S.A.
} 


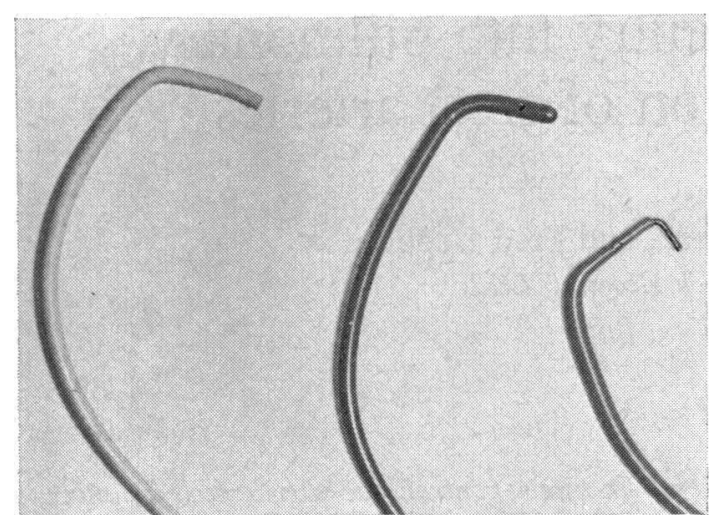

FIG. I Tips of the three catheters described in this paper. On the left is the original Cobra catheter with a single end-hole. On the right is the first modification, with two side-holes and a tip curve retainer in situ. In the centre is the final version of the catheter, blindended, with no taper, and four spirally placed endholes at the tip.

For this reason it was not felt to be essential to enter the pulmonary artery in all patients with transposition.

Two categories were delineated, those in whom entry to the pulmonary artery was regarded as essential, and those in whom it was regarded as desirable. The first category included all patients being assessed for 'corrective' surgery. The second included all infants under 3 months of age not being assessed for corrective surgery whose left ventricular peak systolic pressure (which was measured in all) was greater than 70 per cent of peak systemic arterial systolic pressure.

The original Cobra catheter (Fig. I) had two major disadvantages with respect to intracardiac manipulation, though it was successfully used 5 times. The single end-hole caused difficulties with pressure measurement and sampling, and the polyurethane of which the catheter is made did not slide freely within the vein into which it was placed. The first modification made to the catheter was to make it of polyethylene and add two side holes near the tip ${ }^{1}$ (Fig. I). This modification was used 9 times, until the final version was ready for use (Fig. I). In this the tapered tip and end-hole were abolished and an extra two side holes added in order to allow maximal, yet safe, delivery rates of contrast medium: this version ${ }^{1}$ has been used 5 times. As no significant difference between the three catheters emerged as far as the speed of entry to the pulmonary artery was concerned, the results are presented below as a single group.

The overall catheter shape and the mode of entry into the pulmonary artery are shown in Fig. 2.

The frequency response of the final version of the catheter $(80 \mathrm{~cm}, 6 \mathrm{~F}$ external diameter) connected to a

${ }^{1}$ Available from William Cook (Europe) Ltd., Söborg, Denmark.

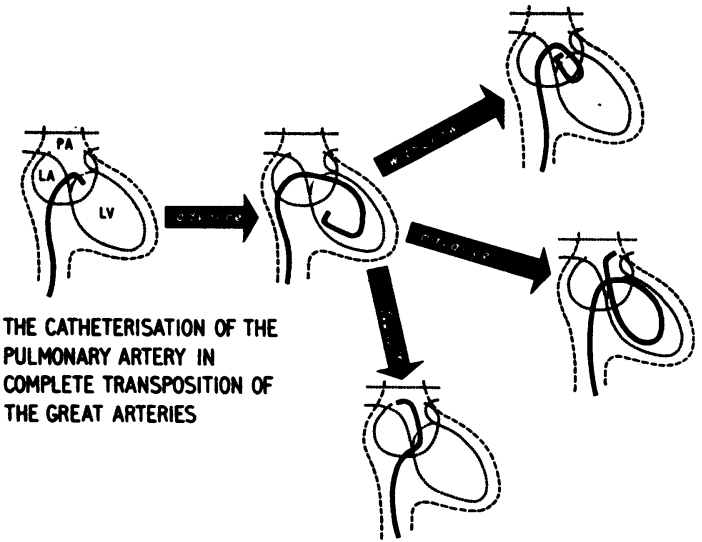

FIG. 2 This demonstrates the mode of entry to the pulmonary artery from the saphenous or femoral vein. The long S-bend of the catheter allows easy passage of the catheter across the interatrial septum and mitral valve into the left ventricle. It also facilitates crossing the interatrial septum when approaching from the axillary vein. Once the catheter is in the left ventricle the manipulation required is the same regardless of the means of approach. The catheter can usually be looped within the left ventricle with ease because it is the tight ' $k$ nuckle' at the end of the catheter rather than the tip which contacts the endocardium. Occasionally it has been necessary to pass the catheter through the mitral valve on a loop made in the left atrium by gentle pressure on a right pulmonary vein/left atrial junction. Once there is a loop in the left ventricle, three alternatives are open:

i) The catheter is withdrawn gently. If the tip can be kept anterior, it will pass through the pulmonary rather than the mitral valve.

ii) The catheter is advanced, with the same result.

iii) The catheter is rotated counterclockwise, whereupon it flicks through the pulmonary valve with great rapidity. This manoeuvre is, of the three, the most often successful. It is undoubtedly assisted by the catheter's built-in torque control.

Statham $23 \mathrm{~Gb}$ transducer was determined in vitro using a varying frequency pressure generator.

In the 2 patients with intact atrial septum a Shirey transvalvular catheter ${ }^{1}$ (Shirey and Sones, 1966) was introduced into the axillary artery and advanced on a loop through the aortic valve and, by keeping the tip posterior, through the ventricular septal defect. When looped in the left ventricle and advanced, the tip passed into the pulmonary artery with ease. This method is frequently successful in patients with single ventricle: however, these are not discussed in this paper.

${ }^{1}$ USCI International Inc., Murray Hill, New Jersey, U.S.A. 


\section{Results}

These are presented for the first 19 uses of the new catheter over a period of 23 months in 17 patients. In 15 catheterizations, entry to the pulmonary artery, was regarded as essential and in 4 desirable. Two patients in the 'essential' category developed transient bradycardia, but the pulmonary artery was entered nevertheless. No other complications occurred, except in I-day-old patient in the 'desirable' category. In this case, the original Cobra catheter induced atrial flutter on entry to the right atrium, so the catheterization was thereupon terminated. At subsequent catheterization 8 months later in the same patient the pulmonary artery was entered.

In the remaining 18 catheterizations the pul-

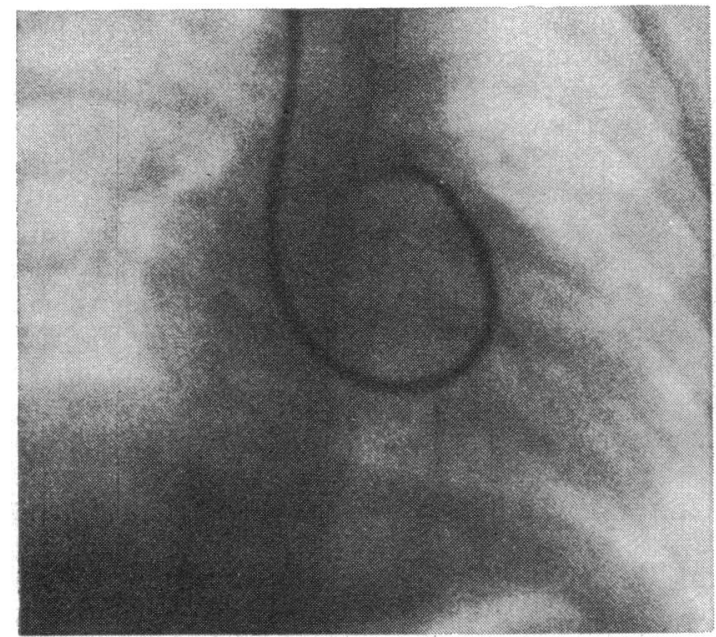

$a$ monary artery was entered from the right atrium each time in a time varying from $40 \mathrm{~s}$ to $15 \mathrm{~min}$ (median $5 \mathrm{~min}$ ). The two longest times (IO and 15 min) were in patients catheterized from the axillary vein which was used 3 times. The femoral vein was used 3 times and the saphenous vein 12 times. Patients' ages ranged from 2 days to 6 years (median 8 months) and weights from 3.1 . to $13.3 \mathrm{~kg}$ (median $6.9 \mathrm{~kg})$. The maximum systolic gradient across the pulmonary valve was $30 \mathrm{mmHg}(4.0 \mathrm{kPa})$. One patient had tricuspid atresia, 4 had a ventricular septal defect, and 4 a persistent ductus arteriosus. In 2 of these the catheter, having entered the pulmonary artery, traversed the ductus. Fig. 3 illustrates the catheter position as seen radiographically. Entry to the right ventricle and aorta presented no

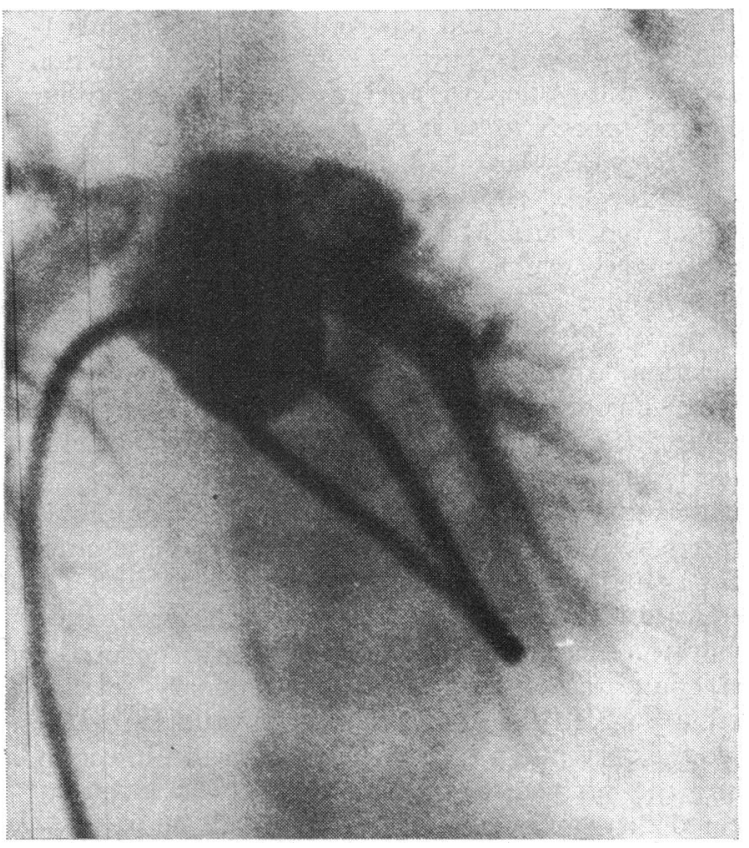

$b$

FIG. 3 a) Single frame of cineangiogram in the frontal view of the blind-ended catheter, immediately before injection of contrast medium, to show catheter position. The catheter had been introduced into the right axillary vein of an 8-month-old patient and advanced across the atrial septal defect, mitral, and pulmonary valves. The ventricular septum was intact. Note the similarity in position to that reached in a non-transposed pulmonary artery.

b) Single frame from frontal plane cineangiogram of 3-week-old patient with $D$-transposition, tricuspid atresia, and an interrupted aortic arch. The patient is rotated somewhat to the right. The blind-ended catheter has been advanced across a patent foramen ovale and the mitral and pulmonary valves into the main pulmonary artery. As the infant had already had two high-dose angiocardiograms this injection was done by hand with $2 \mathrm{ml}$ contrast medium. The descending aorta is seen to opacify via a persistent ductus arteriosus immediately above the take-off of the descending left pulmonary artery. 
problem with this catheter and pulmonary veins were entered with particular ease.

The frequency response of the catheter and transducer was flat \pm 5 per cent to $18 \mathrm{~Hz}$ and an injection rate of $5.9 \mathrm{ml} / \mathrm{s}$ of 70 per cent Conray ${ }^{1}$ can be achieved with an injection pressure of $4 \mathrm{kpm} / \mathrm{cm}^{2}$ through the $6 \mathrm{~F}, 80-\mathrm{cm}$ blind-end catheter.

Entry to the pulmonary artery with the Shirey catheter was achieved in 3 and 10 minutes in 2 patients aged 5 and 13 years.

\section{Discussion}

The very multiplicity of methods advocated for entry to the pulmonary artery in complete transposition indicates that none is entirely satisfactory. A review of the published reports suggests that the Swan-Ganz flow-directed balloon catheter is the most reliable method reported to date, particularly when the tip is curved before its introduction (Jones and Miller, 1973). This new catheter, in our hands, appears to be at least as reliable and as swift as the Swan-Ganz catheter when used by others (Kelly et al., I97I; Stanger et al., 1972; Black, I972; Jones and Miller, 1973), but has additional advantages which relate specifically to its lack of dependence on blood flow. Since it is semi-rigid, it never relies on balloon inflation to maintain its position, and this assumes maximal importance when angiocardiography is attempted. Experience of angiocardiography with the Swan-Ganz catheter has been disappointing (Stanger et al., 1972). It is true that preoperative pulmonary arteriography is rarely required in complete transposition, though Fig. 3b (from a case of tricuspid atresia with Dtransposition and an interrupted aortic arch) provides an example of its value. Comparison of the ascending aortogram with the pulmonary arteriogram enabled the extent of the interruption to be judged very much more reliably than did injection into the left ventricle. Postoperatively, it is anticipated that pulmonary angiography will be particularly valuable in assessing pulmonary venous obstruction after Mustard's operation. But apart from the particular needs for pulmonary arteriography, it is of considerable practical value to be able to use the same catheter that enters the pulmonary artery for angiocardiography, sampling, and pressure measurements in any chamber or vessel. Traversing a persistent ductus arteriosus from pulmonary artery to aorta was achieved in 2 of 4 patients, thus providing valuable information with extreme simplicity.

Use of the catheter does depend upon the

${ }^{2}$ May and Baker Ltd. (Dagenham). presence of an interatrial communication. With the widespread use of atrial septostomy and the trend toward early correction, such a communication will almost always be present. The modification described of the method of retrograde catheterization of the pulmonary artery (Mullins et al., 1972) does provide an alternative solution to the problem of the intact interatrial septum. Apart from the retrograde technique (which depends upon the presence of a ventricular septal defect), and suprasternal puncture (which is not without risk) (Jones and Miller, 1973), all methods described for entry to the pulmonary artery have been from the saphenous or femoral vein. This is impossible if there is thrombosis of the inferior vena cava, which not infrequently occurs after balloon septostomy (Hawker et al., 1971). Even if this does not occur, patients often need recatheterization so frequently that the ability to use this catheter from the axillary vein is a major advantage.

The tapered-tip version of the catheter has been used percutaneously by us for selective injection of collateral arteries in pulmonary atresia by the transarterial route. It could presumably be used transvenously in transposition, but we have not tried this.

The ease with which this catheter entered the pulmonary artery, even in neonates (of which there were 3), encourages us to extend the category of sick neonates in which entry to the pulmonary artery should be attempted to include those in which the left ventricular pressure is less than 70 per cent of systemic arterial pressure. With this in mind, a $5 \mathrm{~F}$ catheter having the same shape as the one described is being manufactured. All in all, it is hoped that this catheter will prove to be of considerable value to anyone involved in investigating patients with complete transposition.

The authors would like to thank Mr. Robert West for his help in construction of the catheters.

\section{References}

Black, I. F. S. (1972). Floating a catheter into the pulmonary artery in transposition of the great arteries. American Heart fournal, 84, 761.

Carr, I., and Wells, B. (I966). Coaxial flow-guided catheterization of the pulmonary artery in transposition of the great arteries. Lancet, 2, 318.

Celermajer, J. M., Venables, A. W., and Bowdler, J. D. (1970). Catheterization of the pulmonary artery in transposition of the great arteries. Circulation, 4r, 1053.

Hawker, R. E., Celermajer, J. M., Cartmill, T. B., and Bowdler J. D. (I97I). Thrombosis of the inferior vena cava following balloon septostomy in transposition of the great arteries. American Heart fournal, 82, 593.

Jones, S. M., and Miller, G. A. H. (1973). Catheterization of the pulmonary artery in transposition of the great arteries using a Swan-Ganz flow-directed catheter. British Heart fournal, 35, 298. 
Kelly, D. T., Krovetz, L. J., and Rowe, R. D. (197I). Doublelumen flotation catheter for use in complex congenital cardiac anomalies. Circulation, 44, 910.

Mullins, C. E., Neches, W. H., Reitman, M. J., El-Said, G., and Riopel, D. A. (1972). Retrograde technique for catheterization of the pulmonary artery in transposition of the great arteries with ventricular septal defect. American Fournal of Cardiology, 30, 385.

Pickering, D., McDonald, P., and Kidd, B. S. L. (1971). Catheterization of the pulmonary artery in transposition of the great arteries: a new technique. Pediatrics, 47, 1068.

Rahimtoola, S. H., Ongley, P. A., and Swan, H. J. C. (1966). Percutaneous suprasternal puncture (Radner technique) of the pulmonary artery in transposition of the great vessels. Circulation, 33, 242.
Shirey, E. K., and Sones, F. M., Jr. (1966). Retrograde transaortic and mitral valve catheterization. American fournal of Cardiology, 18, 745.

Stanger, P., Heymann, M. A., Hoffman, J. I. E., and Rudolph, A. M. (1972). Use of the Swan-Ganz catheter in cardiac catheterization of infants and children. American Heart Fournal, 83, 749.

Tynan, M. (1972). Transposition of the great arteries. Changes in the circulation after birth. Circulation, 46, 809.

Requests for reprints to Dr. F. J. Macartney, Killingbeck Hospital, York Road, Leeds LSI4 6UQ. 